\title{
Microestructura y propiedades mecánicas de dos aceros para herramientas con ultra alto contenido de boro
}

\author{
J.A. Jiménez ${ }^{(*)}$, P. Acosta ${ }^{(* *)}$, G. Frommeyer ${ }^{(* *)}$ y O.A. Ruano ${ }^{(*)}$
}

Resumen En el presente trabajo se han modificado dos aceros para herramientas convencionales por la presencia de un contenido de boro entre 0,5 y $1 \%$ en masa. Ambos aceros se procesaron por la ruta pulvimetalúrgica, incluyendo atomización por argón y compactación isostática en caliente. El material compactado presentó una microestructura caracterizada por una distribución de partículas de borocarburos $M_{23}(C, B)_{6}$ fina y homogénea en una matriz de ferrita-martensita. Esta microestructura permanece prácticamente inalterada tras los ensayos de cambios en la velocidad de deformación durante el ensayo de compresión a temperaturas entre 750 y $1.000{ }^{\circ} \mathrm{C}$. En el caso del acero Fe-1B-1C se obtuvo un valor para el exponente de la tensión de 4,5, que sugiere que la deformación plástica está controlada por un mecanismo de fluencia por movimiento de dislocaciones. Por otro lado, para el acero Fe-0,5B-1,5C, se obtuvieron valores para el exponente de la tensión comprendidos entre 2 y 3 , que sugieren que la deformación plástica está controlada por un mecanismo de deslizamiento de fronteras de granos. En ambos casos, se encontró un valor de la energía de activación para la deformación plástica que se asoció con la energía de autodifusión del hierro a través de la red.

Palabras clave: Aceros con ultra alto contenido de boro. Aceros para herramientas. Fluencia.

\section{Microstructure and mechanical properties of two tool steels with ultrahigh boron content}

\begin{abstract}
In the present work, two selected tool steels have been modified by a boron addition of 0.5 and 1 mass $\%$. Both steels were processed by powder metallurgy methods, including argon atomization and hot isostatic pressing. The consolidated materials presented a microstructure consisting of a fine and homogeneous distribution borocarbides $\mathrm{M}_{23}(\mathrm{C}, \mathrm{B})_{6}$ in a ferrite-martensite matrix. No changes are observed in the microstructure after deformation by compression-strain-rate-change tests at temperatures ranging from 700 to $1,100{ }^{\circ} \mathrm{C}$. For the Fe-1B-1C steel, a stress exponent of 4.5 was obtained, that suggests that slip creep is the controlling deformation mechanism. On the other hand, a stress exponent between 2 and 3 was obtained for the Fe-0.5B-1.5C steel that suggests that grain boundary sliding is the controlling deformation mechanism. In both cases, the activation energy for creep was related to the activation energy for iron self-diffusion.
\end{abstract}

Keywords: Ultrahigh boron steels. Tool steels. Creep.

\section{INTRODUCCIÓN}

Un acero de elevadas prestaciones debe presentar altas características de resistencia, dureza y tenacidad acompañadas de buena resistencia al des-

(*) Centro Nacional de Investigaciones Metalúrgicas, CENIM (CSIC), Avda. de Gregorio del Amo 8. 28040Madrid (España).

(**) Max Planck Institut für Eisenforschung GmbH, Max Planck Strasse 1. 40237-Düsseldorf (Alemania). gaste. En el caso de los aceros al carbono, estas propiedades se basan en su endurecimiento mediante la transformación martensítica. El problema que se presenta se debe a la progresiva pérdida de propiedades con la exposición a temperaturas intermedias. La ruta tradicional de solventar este problema es a través de una fina precipitación de carburos a altas temperaturas, que se conoce como segunda precipitación. Un procedimiento alternativo consistiría en proporcionar al acero una elevada fracción 
en volumen ( $>20 \%$ vol.) de finas y duras partículas insolubles de segunda fase homogéneamente dispersadas en la matriz, como pueden ser carburos o boruros. Además, en el caso de los aceros al boro, como los boruros son insolubles (1 y 2) y sólo coalescen a muy elevadas temperaturas, se espera que estos aceros conjuguen la resistencia al desgaste con una elevada resistencia a temperatura media y alta (3).

Con esta idea como punto de partida, se han modificado dos aceros para herramientas introduciendo un contenido en boro entre 0,5 y $1 \%$ en masa. El boro en combinación con el carbono debería proporcionar partículas de borocarburos $\mathrm{M}_{23}(\mathrm{CB})_{6}$, disminuyéndose así la cantidad de carbono que puede disolverse en la matriz (4). Debido a la escasa solubilidad del boro en la matriz austenítica, para conseguir una distribución fina y homogénea de estas partículas se recurrió a la atomización por argón y consolidación de los polvos mediante la técnica de compactación isostática en caliente (CIC) (5 y 6). En el presente trabajo, se analiza la microestructura y el comportamiento mecánico a baja y alta temperatura de los compactos resultantes.

\section{PROCEDIMIENTO EXPERIMENTAL}

En el presente estudio se han preparado por atomización con argón dos aceros de herramientas con ultra alto contenido de boro y carbono. La composición química de dichos aceros se presenta en la tabla I.

Los polvos resultantes de la atomización se encapsularon y desgasificaron en vacío a $400{ }^{\circ} \mathrm{C}$ durante $2 \mathrm{~h}$. El material encapsulado se sometió a un ciclo de compactación isostática en caliente a una presión de $180 \mathrm{MPa}$ durante $2 \mathrm{~h}$ a 1.000 y $1.100{ }^{\circ} \mathrm{C}$ para las aleaciones Fe-1B-1C y $\mathrm{Fe}-0,5 \mathrm{~B}-$ $1,5 \mathrm{C}$, respectivamente. Se obtuvieron compactos de ambos aceros con una porosidad inferior al $1 \%$.

Para el estudio microestructural de los compactos, se utilizaron técnicas de difracción de rayos $\mathrm{X}$, microscopía óptica y electrónica de barrido (MEB). Las propiedades mecánicas se determinaron mediante ensayos de compresión sobre probetas prismáticas de $4 \times 4 \mathrm{~mm}$ de base y $6 \mathrm{~mm}$ de altura. A partir de ensayos con cambios en la velocidad de deformación, entre $3 \times 10^{-5}$ y $10^{-2} \mathrm{~s}^{-1}$, durante el ensayo de compresión a $750-1.000{ }^{\circ} \mathrm{C}$, es posible tanto el cálculo del exponente de la tensión como la energía de activación del proceso.

\section{RESULTADOS}

\subsection{Microestructura}

La microestructura presente en los polvos de partida de ambas aleaciones es similar. A modo de ejemplo, la figura 1 muestra la microestructura de una partícula de polvo del acero Fe-1B-1C. Se observa una microestructura dendrítica, en la que la fase primaria de solidificación aparece rodeada de una red de partículas del borocarburos $\mathrm{M}_{23}(\mathrm{C}, \mathrm{B})_{6}$. De acuerdo con el diagrama de difracción de rayos $\mathrm{X}$, la fase primaria de solidificación corresponde a ferrita para el acero Fe-1B-1C y austenita para el acero $\mathrm{Fe}-0,5 \mathrm{~B}-1,5 \mathrm{C}$.

Después de la compactación isostática en caliente a $1.000^{\circ} \mathrm{C}$ para el acero $\mathrm{Fe}-1 \mathrm{~B}-1 \mathrm{C}$ y a $1.100{ }^{\circ} \mathrm{C}$ para el acero $\mathrm{Fe}-0,5 \mathrm{~B}-1,5 \mathrm{C}$ se obtiene una microestructura más gruesa compuesta, en ambos casos, por una fina dispersión de partículas de segunda fase en una matriz ferrítica, como se muestra en la figuras $2 a$ y $2 b$. Estas partículas de segunda fase fueron identificadas, mediante difracción de rayos

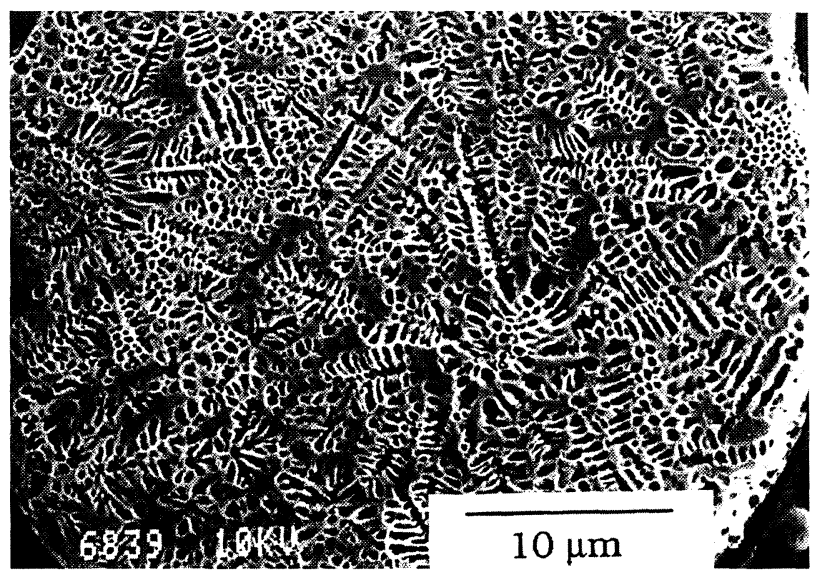

Fig. 1.- Micrografía de una partícula de polvo del acero $\mathrm{Fe}-1 \mathrm{~B}-1 \mathrm{C}$.

FIG. 1.- Micrograph of a powder particle of the Fe-1B-1C steel.

TABLA I.- Composición química de los aceros estudiados (\% en masa)

TABLE I.-Chemical composition of the studied steels (mass \%)

\begin{tabular}{|l|c|c|c|c|c|c|c|c|c|}
\hline ACERO & B & Cr & Mo & V & W & Si & Mn & O & C \\
\hline Fe-1B-1C & 1,0 & 5,4 & & 5,7 & 5 & 0,4 & 0,5 & 0,03 & 1,24 \\
Fe-0,5B-1,5C & 0,5 & 1,2 & 1,2 & 1,3 & & 0,3 & 0,6 & 0,02 & 1,59 \\
\hline
\end{tabular}



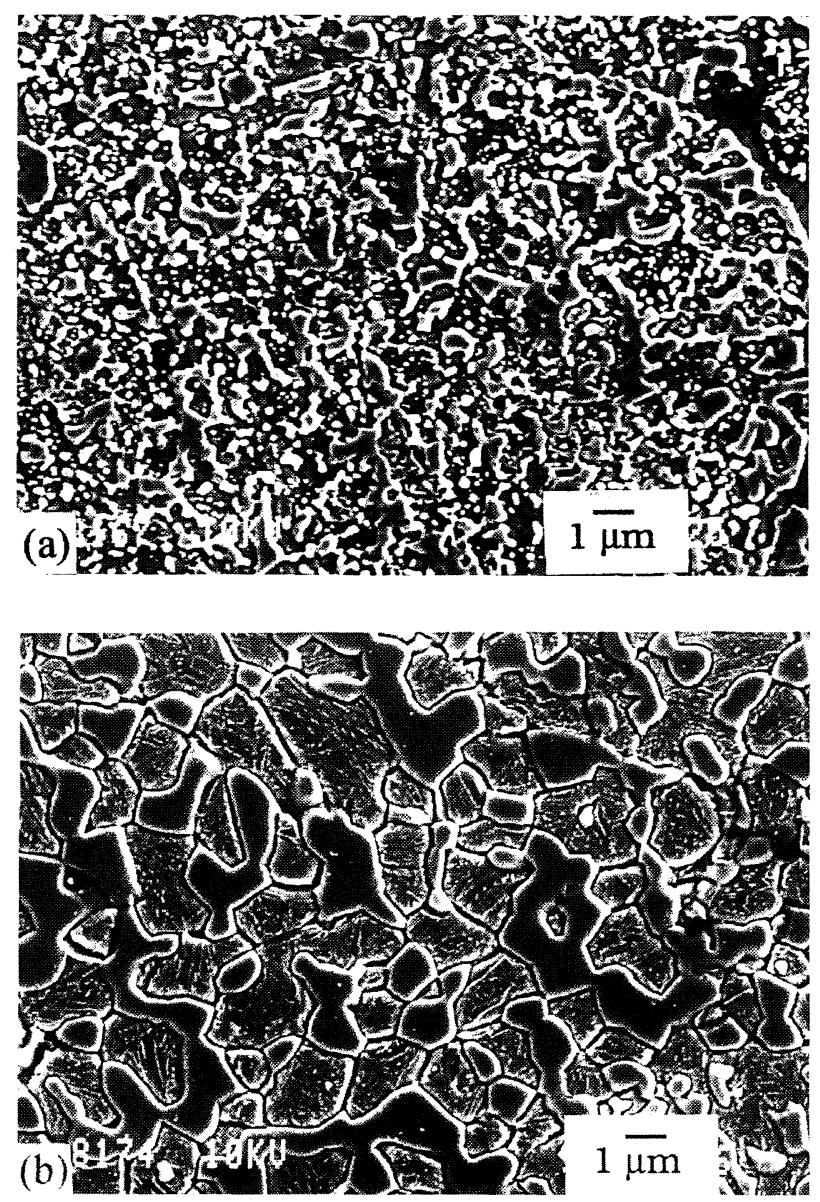

FIG. 2.- Microestructura después de la compactación isostática en caliente. a) a $1.000{ }^{\circ} \mathrm{C}$ para el acero Fe-1B-1C y $b$ ) a $1.100{ }^{\circ} \mathrm{C}$ para el acero $\mathrm{Fe}-0,5 \mathrm{~B}-1,5 \mathrm{C}$.

FIG. 2.- Microstructure after hot isostatic pressing a) at $1,000{ }^{\circ} \mathrm{C}$ for the $\mathrm{Fe}-1 \mathrm{~B}-1 \mathrm{C}$ steel, and b) at $1,100^{\circ} \mathrm{C}$ for the $\mathrm{Fe}-0,5 \mathrm{~B}-1,5 \mathrm{C}$ steel.

$\mathrm{X}$, como $\mathrm{M}_{23}(\mathrm{C}, \mathrm{B})_{6}, \mathrm{MC}$ y $\mathrm{M}_{3} \mathrm{~B}_{2}$, para el acero Fe1B-1C, y $\mathrm{M}_{23}(\mathrm{C}, \mathrm{B})_{6}$, para el acero Fe-0,5B-1,5. Por otro lado, el diagrama de difracción mostró un achatamiento de los picos de la fase ferrítica, que se asoció con la presencia de martensita, dado que este tipo de aceros templan al aire. Además, se observó disminución de la dureza después de dos recocidos sucesivos a $650^{\circ} \mathrm{C}$.

\subsection{Comportamiento mecánico}

El comportamiento mecánico en el intervalo de temperaturas comprendido entre 750 y $1.100{ }^{\circ} \mathrm{C}$ puede observarse en las curvas de velocidad de deformación real, $\dot{\varepsilon}$, en función de la tensión real corregida por el módulo de Young, $\sigma / E$, que se presentan en la figuras $3 a$ y $3 b$ para los aceros Fe-1B$1 \mathrm{C}$ y Fe-0,5B-1,5C, respectivamente. El módulo de
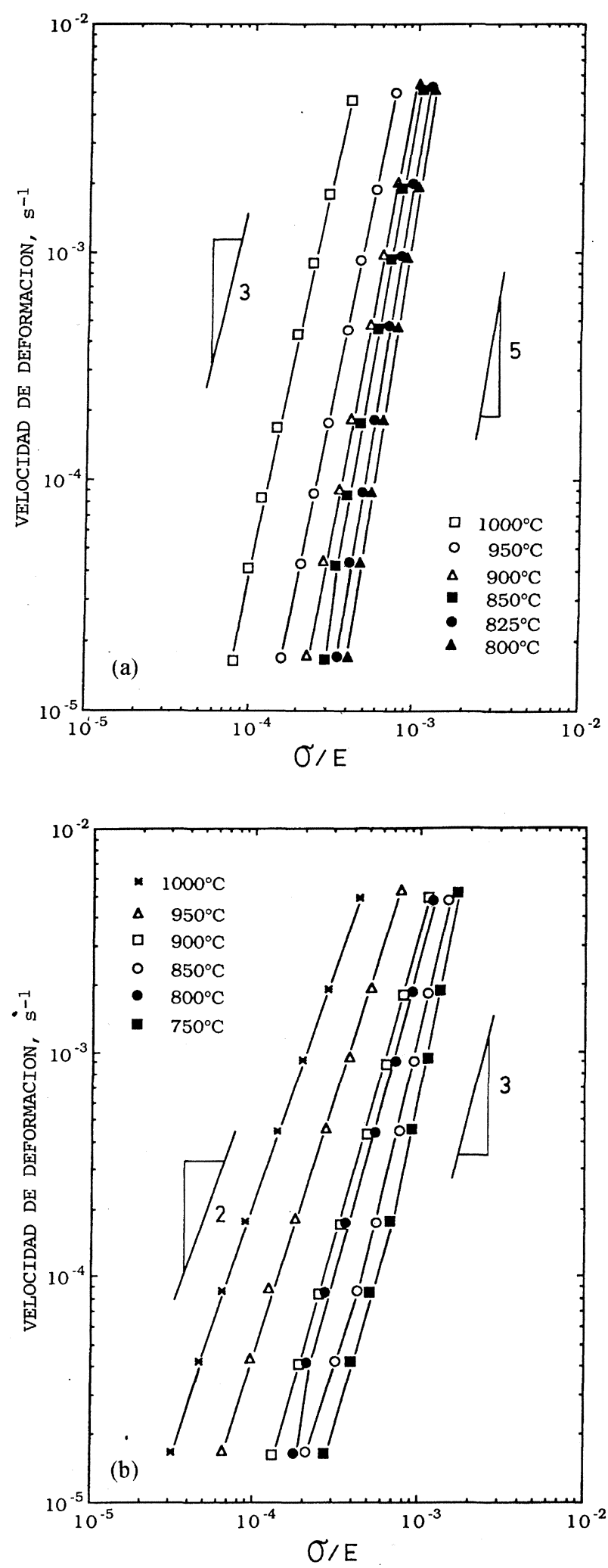

FIG. 3.- Velocidad de deformación en función de la tensión de fluencia corregida por el módulo de Young para $a$ ) el acero Fe-1B-1C y $b$ ) el acero $\mathrm{Fe}-0,5 \mathrm{~B}-1,5 \mathrm{C}$.

FIG. 3.- The strain rate as a function of Young's modulus compensated stress for a) the Fe-1B-1C steel and b) the Fe-O.5B-1.5C steel. 
Young a las temperaturas de ensayo se tomó de Köster (7). Como puede apreciarse en la figura 3, en el caso del acero Fe-1B-1C se obtuvo un valor para el exponente de la tensión de 4,5, mientras que para el acero Fe-0,5B-1,5C se obtuvieron valores comprendidos entre 2 y 3 . Por otro lado, en las figuras $3 a$ y $3 b$ se observa, para un valor constante de $\sigma / E$, un aumento de la velocidad de deformación conforme se incrementa la temperatura de ensayo. Sin embargo, alrededor de la temperatura de transformación ferrita-austenita, la diferencia entre las velocidades de deformación es menor en acero $\mathrm{Fe}$ $1 \mathrm{~B}-1 \mathrm{C}$ y, en el caso del acero Fe-0,5B-1,5, el material fluye a $850^{\circ} \mathrm{C}$ a una velocidad inferior que a $800^{\circ} \mathrm{C}$.

\section{DISCUSIÓN}

Los resultados de los ensayos de compresión con cambios en la velocidad de deformación presentados en las figuras $3 a$ y $3 b$ se analizaron de acuerdo con la ecuación constitutiva de la fluencia:

$$
\dot{\varepsilon}=A \exp \left(\frac{-Q_{c}}{R T}\right)\left(\frac{\sigma}{E}\right)^{n}
$$

donde $A$ es una constante que depende del material, $Q_{\mathrm{c}}$ es la energía de activación de fluencia, $R$ es la constante de los gases y $T$ la temperatura absoluta.

De acuerdo con la ecuación anterior, la energía de activación para la fluencia puede obtenerse de los datos representados en las figuras $3 a$ y $3 b$ a partir de una representación en escala semilogarítmica de $\dot{\varepsilon}$ frente al inverso de la temperatura absoluta de ensayo para un valor constante de $\sigma / E$. En las figuras $4 a$ y $4 b$ se presenta la energía de activación a $\sigma / E=5 \times 10^{-4}$ para los aceros Fe-1B-1C y $\mathrm{Fe}-0,5 \mathrm{~B}-1,5 \mathrm{C}$, respectivamente. Se observa, en ambos casos, una discontinuidad a la temperatura de transformación ferrita-austenita, (alrededor de 900 y $850^{\circ} \mathrm{C}$ para los aceros $\mathrm{Fe}-1 \mathrm{~B}-1 \mathrm{C}$ y $\mathrm{Fe}-0,5 \mathrm{~B}-$ $1,5 \mathrm{C}$, respectivamente). Esta discontinuidad se asocia con una resistencia a fluencia distinta en ambas fases.

Para temperaturas de ensayo por encima de la $A_{1}$, la figura 4 presenta valores para la energía de activación de 280 y $300 \mathrm{~kJ} / \mathrm{mol}$, similares al valor de la energía de activación para la difusión a través de la fase austenítica $(270 \mathrm{~kJ} / \mathrm{mol})$ (8). Dado que el exponente de la tensión es del orden de 4,5 para el acero $\mathrm{Fe}-1 \mathrm{~B}-1 \mathrm{C}$, su comportamiento a fluencia podría explicarse mediante la ecuación fenomenológica para la fluencia por movimiento de dislocaciones (9):

$$
\dot{\varepsilon}=10^{11}\left(D_{R} / b^{2}\right)(\sigma / E)^{5}
$$
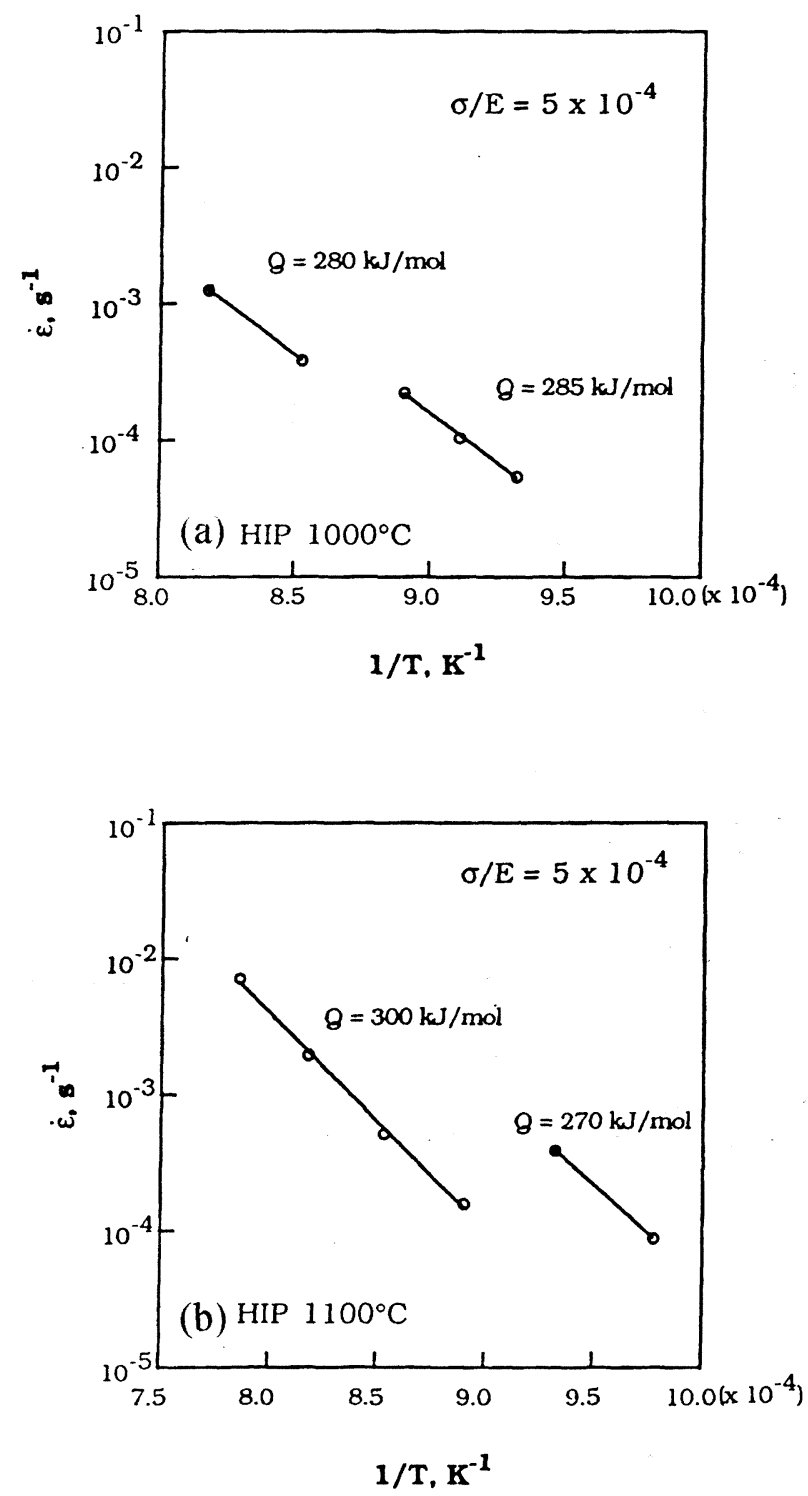

FIG. 4.- Energías de activación para la deformación para $a$ ) el acero $\mathrm{Fe}-1 \mathrm{~B}-1 \mathrm{C}$ y $b$ ) el acero $\mathrm{Fe}-0,5 \mathrm{~B}-1,5 \mathrm{C}$.

FIG. 4.- Activation energies for a) the Fe-1B-1C steel and $b$ ) the Fe-0.5B-1.5C steel.

donde $D_{\mathrm{R}}$ es el coeficiente de difusión a través de la red y $b$ el vector de Burgers. Si se introducen en esta expresión los datos para la autodifusión del hierro en la fase austenítica, se encuentra un razonable acuerdo entre los valores experimentales y los que se deducen de la ec. [2].

Por otro lado, para el acero Fe-0,5B-1,5C, se obtuvo un exponente de la tensión cercano a 2 , de modo que el mecanismo de deformación puede asociarse a un deslizamiento de fronteras de grano. La ecuación fenomenológica que se utiliza en este caso es (9):

$$
\dot{\varepsilon}=6,4 \times 10^{9}\left(D_{R} / d^{2}\right)(\sigma / E)^{2}
$$


donde $d$ es el tamaño de grano. En este caso, los valores de la velocidad de deformación que se predicen son un factor 3 superiores a los que se encontraron experimentalmente.

La energía de activación que se deduce para temperaturas por debajo de la $A_{1}$, alrededor de 280 $\mathrm{kJ} / \mathrm{mol}$, es superior, para ambos aceros, a la energía para la autodifusión del hierro a través de la red ferrítica $(239 \mathrm{~kJ} / \mathrm{mol})$. Este elevado valor de la energía de activación hace pensar que la deformación está controlada por la difusión a través de la red. Esta red podría ser la del borocarburo, ya que la elevada fracción de volumen de esta fase (alrededor del $40 \%$ ) puede condicionar la deformación. Sin embargo, no hay datos suficientes de difusión en la bibliografía para corroborar esta hipótesis.

\section{CONCLUSIONES}

- El procesado de dos aceros para herramientas con ultraalto contenido de boro mediante la ruta pulvimetalúrgica, incluyendo atomización por argón y compactación isostática en caliente (a 1000 y $1100^{\circ} \mathrm{C}$ para las aleaciones Fe-1B-1C y Fe-0,5B-1,5C, respectivamente) permite la obtención de una microestructura caracterizada por una fina y homogénea distribución de partículas de borocarburos $\mathrm{M}_{23}(\mathrm{CB})_{6}$ en una matriz de ferrita-martensita.

- Para el acero Fe-1B-1C, se obtuvo, a temperaturas por encima de la $A_{1}$ un exponente de la tensión próximo a 5 y una energía de activación para la fluencia similar a la energía de activación para la autodifusión de hierro en austenita. A estas temperaturas, estos resultados sugieren que la deformación plástica está controlada por el movimiento de dislocaciones. Además, se encontró concordancia entre los valores experimentales de la velocidad de deformación y los predichos a partir de una ecuación fenomenológica.

- Para el acero Fe-0,5B-1,5C se obtuvo, a temperaturas por encima de la $A_{1}$, un exponente de la tensión próximo a 2 y una energía de activación para la fluencia similar a la energía de activación para la autodifusión de hierro en austenita A estas temperaturas, estos resultados sugieren que la deformación plástica está controlada por el deslizamiento de fronteras de grano. Además, se encontró concordancia entre los valores experimentales de la velocidad de deformación y los predichos a partir de una ecuación fenomenológica.

- Para ambos aceros se encontró, para temperaturas por debajo de la $A_{1}$, un valor para la energía de activación superior a la energía para la autodifusión del hierro a través de la red ferrítica, que podría indicar que la elevada fracción de volumen de borocarburos puede condicionar la deformación.

\section{Agradecimiento}

Los autores agradecen la financiación de este trabajo por la CICYT (Proyecto MAT 97-0695C02-01).

\section{REFERENCIAS}

(1) Busby, P.E., Warga, M.E. y Wells, C. J. Met. 5, 1953: 1.463-1.468.

(2) GOLDSCHMidT, H.J. J. Iron Steel Inst., 209, 1971: 900-909.

(3) Ray, R., Panchanathan, V. y Isserow, S. J. Met. Jun. 1983: 30.

(4) Acosta, P., Jiménez, J.A., Ruano, O.A. y Frommeyer, G. Steel Res. 66, 1995: 360-365.

(5) Jiménez, J.A., Gonzalez-Doncel, G. y Ruano, O.A. Metall. Mater. Trans. A, 27, 1996: 1861.

(6) JimÉnez, J.A., Gonzalez-Doncel, G. y Ruano, O.A. $A d v$. Mater. 7, 1995: 130.

(7) Köster, W. Z. Metallkde., 39, 1948:1.

(8) Frost, H.J. y Ashby, M.F. Deformation-Mechanism Maps, p. 60; 1982, Oxford (R.U.), Pergamon Press.

(9) Ruano, O.A. y Sherby, O.D. Rev. Phys. Appl. 23, 1988: 625 . 